CRÍTICA, Revista Hispanoamericana de Filosofia

Vol. XXI, No. 61 (abril 1989): 103-116

\title{
DISCUSIONES
}

SIGNIFICADO LITERAL: ENTRE LA PROFUNDA NECESIDAD DE UNA CONSTRUCCIÓN Y LA MERA NOSTALGIA *

Julio Cabrera Universidad de Brasilia

En mi artículo "Cortando árboles y relaciones" (Crítica, vol. XVI, núm. 46, México, abril 1984) traté de mostrar que la noción de "significado literal" de un término, entendido como invariable a través de sus ocurrencias, es - como cualquier otro término unificador u homogeneizante- "proyectado" por cierta teoría del lenguaje y no -como lo sugiere cierto "modo material de hablar" muy usual- descubierto en el mundo. Partía en ese trabajo de la intuición fundamental de que toda y cualquier "invariancia" debe ser proyectada sobre el material lingüístico y de que toda teoría opera, fundamentalmente, de una manera unificadora. Con esto, estaba yo planteando una cuestión epistemológica de carácter fundamental, vinculada con la construcción de conceptos.

Marcelo Dascal, en su artículo "Una crítica reciente a la noción de significado literal" (p. 35, nota 2), interpreta que yo formo parte del ejército de autores que discuten acerca de los problemas de delimitación entre semántica

- Una réplica a $M$. Dascal, "Una crítica reciente a la noción de significado literal”, Crítica, vol. XVIII, núm. 53, México, agosto 1986. 
y pragmática, literalismo y contextualismo y otras problemáticas demasiado "actuales" para mi gusto un tanto "unzeitgemässig" ( $c f r$. mi "A Lógica condenada. Uma abordagem extemporânea de filosofia da Lógica". Hucitec, São Paulo, Brasil, 1987), y a la luz de esas cuestiones en disputa, me clasifica sin piedad entre los "contextualistas": "Cabrera saca la conclusión de que yo conservo la noción de significado literal nada más por nostalgia, y adopta en cambio una posición contextualista, según la cual [citándome] 'los significados de los términos son co-constituidos en y por el entorno respectivo' en forma 'dinámica'". Soy claramente culpable de la acusación de "contextualismo". Por lo menos a causa de dos afirmaciones de mi artículo del 84, una de ellas la ya citada por Dascal (p. 21 de mi artículo), y la otra en la p. 27: "Las condiciones de verdad de un enunciado no podrán formularse sin antes examinar cómo se constituye la significación de una palabra en un contexto dado...". En estas afirmaciones poco cuidadosas desconozco la fuerza de mi propia tesis, y ellas mismas caen bajo lo que critico, o sea, el modo material de hablar que se adopta cuando se dice algo sobre significaciones. Pues del desarrollo de mi artículo debe necesariamente desprenderse que tanto los "significados literales" como los "significados contextuales" son -en la medida en que pretenden unificar material lingüístico diverso - igualmente proyectados por la teoría, y precisamente ése es el motivo por el cual mi posición no solamente no puede ser "contextualista", sino que la totalidad de mi argumentación se mueve en un nivel completamente diferente de aquel en el que discuten contextualistas y literalistas, moderados o inmoderados.

Dascal cae, en su réplica, en una trampa ya anticipada en mi artículo. Me cito: “... ese modo material de hablar prepara una trampa para todo aquel que discuta la idea de Searle - como es mi caso aquí- pues 
se podría pensar que lo que yo quiero demostrar es algo así como que 'no existe' significado literal, o que 'no hay nada' a lo que pueda llamarse significado común a varias ocurrencias de un mismo término, etc." ("Cortando árboles...", p. 18). Él ha definido el "contextualismo" precisamente en el modo material de hablar: "He denominado 'contextualismo'... a la tesis de que las expresiones lingüísticas carecen de un significado 'literal' establecido por las reglas del lenguaje, adquiriendo sus significados exclusivamente en función del contexto en el que son empleadas" ("Una crítica reciente...", p. 34, subrayado mío). Claro que no soy "contextualista" en el sentido así definido, pues he escrito en la misma página antes citada: "Pero aunque mi argumentación apunta efectivamente contra la idea de significado literal como algo que diferentes ocurrencias de términos tendrian de común, jamás negaré aquí que podemos construir teorías del lenguaje con significados literales, y que pueden ser interesantes, útiles y perfectamente bien estructuradas. Lo que aquí se sostendrá no será, ciertamente, una teoría ontológica". En la versión material usual tanto el literalismo como el contextualismo son tesis ontológicas en este sentido. Toda mi argumentación va dirigida NO contra el literalismo o el contextualismo, sino contra ese proceso de ontologización de una teoría del lenguaje.

La trampa consiste en confundir dos tesis de nivel diferente: T1) No hay algo como un significado literal. T2) No es necesario construir algo como un significado literal. Yo me comprometo, claro está, con T2 y no con T1. (De hecho, ino tengo posición definida respecto de las polémicas entre literalistas y contextualistas!) No creo que los significados literales sean cosas, creo que son conceptos, que son construidos como cualquier otro concepto. Así, yo NO discuto que SI ese concepto es construido (y es perfectamente construible) ENTON- 
CES él cumple con todas las funciones que Dascal expone en sus textos. Lo que discuto es la necesidad de una construcción, y no una cuestión de percepción de fenómenos, mundanos o mentales, a través de una exploración empírica del universo. Los significados literales - como muchas otras construcciones teóricas- podrían ser recursos útiles para hablar, comunicar y analizar el lenguaje $\mathrm{y}$, al mismo tiempo, podrían ser teóricamente innecesarios. Quiero mostrar esa no necesidad de dos maneras: una, a través dẹ un test, y otra, a través de consideraciones teóricas más generales.

Para hacer lo primero, es suficiente con insistir en lo dicho en la p. 23 de mi artículo: "Imaginen que tenemos sentencias como las siguientes: 'Abrió los brazos cuando me vio', 'Abrió el coco de un martillazo', 'Abrió una ranura en la madera'. La determinación correcta (y suficiente) de las condiciones de verdad de cada una de ellas puede no basarse en ningún rasgo común, sino precisamente en sus rasgos diferenciadores. Si un niño me pregunta qué significa abrir un coco, no sería extraño responderle: '¿Recuerdas cuando el tío Andrés abrió los brazos al bajar del avión? Pues bien, debes hacer con el coco algo semejante a lo que él hizo con los brazos'. Es obvio que no necesito decir eso. $Y$ tal vez sea aconsejable no decirlo para hacer una determinación correcta de las condiciones de verdad en cada caso. Puedo decir: alguien abre los brazos si los separa del cuerpo, extendiéndolos; alguien abre un coco si consigue partirlo mediante un instrumento suficientemente pesado; alguien abre una ranura si perfora un pedazo de materia permitiendo la visión. Todo el mundo entiende que he dado las condiciones en las que cada uno de esos enunciados es verdadero $\mathrm{y}$, sin embargo, no he tenido que repetir ningún concepto 'común'. Eso no quiere decir que alguien no pudiese responder: alguien abre los brazos cuando los separa del 
cuerpo, alguien abre un coco cuando lo separa en partes mediante el uso de un instrumento, alguien abre una ranura cuando separa las partes de una materia permitiendo la visión. Pero aquí no se quiere probar que eso sea imposible, lo que se quiere probar es que no es necesario" ("Cortando árboles...", p. 23). Considero este texto definitivamente claro, pero, para reforzar, supongan el siguiente test. Se entrega a un grupo de personas una Hoja núm. 1, con las siguientes oraciones:

Hoja 1

1. Juan calentó agua, la colocó en cierto recipiente y puso café dentro del mismo.

2. Lucía acomodó sus ropas y demás efectos personales y los metió dentro de las valijas.

3. Estaba releyendo las diferentes partes de mi discurso, memorizándolas, corrigiéndolas, cuando ella llegó.

4. No me dio tiempo para pensar, meditar y emitir mi respuesta.

5. Los obreros ya habían extraído sus herramientas de las cajas, las habían afilado y limpiado convenientemente.

6. Los niños no estaban vestidos, lavados y con sus útiles en las manos para ir a la escuela.

7. La propuesta le llegó en un momento en el que él no estaba en condiciones psicológicas para analizarla.

8. Les fue anticipando algunas informaciones para darles la gran noticia.

9. Lo reprobaron cuando ya había comprado alimentos, bebidas y disfraces para la fiesta.

10. La obra salió mal porque no la habían ensayado, repetido, etc., un número suficiente de veces. 
A continuación, se pide al grupo que formule las condiciones de verdad de las oraciones 1-10, o sea, las condiciones bajo las cuales esas oraciones pueden decirse con verdad o falsedad. Esto es, claramente, una manera de pedir una interpretación de la significación de esas oraciones. El grupo podrá elegir, para responder esta cuestión, dos líneas de acción diferentes. Una parte podrá referirse a esas condiciones utilizando las diversas acciones que aparecen en 1-10: colocar, meter, pensar, meditar, releer, lavarse, vestirse, estar en condiciones psicológicas de, dar informaciones, comprar alimentos y ensayar, entre otras muchas. Pero no se descarta en absoluto que alguien prefiera dar esas condiciones unificando toda esa diversidad de acciones en una acción "común a todas ellas", en una lista del siguiente tipo:

Hoja 2

1. Juan preparó café.

2. Lucía preparó sus valijas.

3. Estaba preparando mi discurso cuando ella llegó.

4. No me dio tiempo para preparar mi respuesta.

5. Los obreros ya habían preparado sus herramientas.

6. Los niños no estaban preparados para ir a la escuela.

7. La propuesta le llegó en un momento en el que él no estaba preparado para analizarla.

8. Los preparó para darles la gran noticia.

9. Lo reprobaron cuando ya tenía preparada la fiesta.

10. La obra salió mal porque no la habían preparado suficientemente.

¿Quién no ve que esto es una opción, que quien la elige no podría sostener que hay un significado común a 1-10, y que ese significado común debe ser utilizado 
para proporcionar las interpretaciones de esas oraciones? ¿Cómo es posible no ver aún que ese significado común es una construcción del hablante, de acuerdo con su capacidad imaginativa y su dominio particular del idioma? $\mathrm{Si}$ el usuario no construye esa "invariancia", jamás la "descubrirá" en el lenguaje, y construirla es una opción (hasta que alguien demuestre la necesidad - y no tan sólo la posibilidad, jamás discutida - de construirla). Yo sería "contextualista" si sostuviera que el test debe necesariamente responderse manteniendo la multiplicidad de cotextos y prohibiendo responderlo mediante la elaboración de listas del tipo de la Hoja 2. Pero no soy ni literalista ni contextualista, porque pienso que ninguna de esas respuestas es necesaria, sino que se trata de opciones que dependen de los objetivos que cierta teoría del lenguaje esté persiguiendo en cada caso. $\mathrm{Si}$ yo tuviera una postura en esta contienda, seguramente sería a favor de un pluralismo de teorías del lenguaje, donde cada una de ellas - o cada conjunto de teorías de cierto tipo- fuera libre de construir significados literales, contextos, o cualquier otra categoría que fuese útil y conveniente para alcanzar los objetivos propuestos. $\mathrm{Si}$, en cambio, se trata de tesis ontológicas, las teorías del lenguaje quedan sujetas a "constraints" de carácter prohibitivo: si Dascal tiene razón, está prohibido, entonces, construir teorías del lenguaje sin significados literales, en las cuales la interpretación de oraciones se haga a través del puro estudio de contextos; pero si, por ejemplo, Gibbs tiene razón (cfr. "Understanding and Literal Meaning"), entonces está prohibido construir teorías del lenguaje con significados literales, donde las interpretaciones se realicen definiendo ciertos puntos de referencia que funcionen como selectivos de elementos contextuales pertinentes. Claro que yo estaría, en este caso, de acuerdo 
con la instauración de una "Paz Perpetua" entre teorias del lenguaje.

La tentativa de ontologizar nociones engendra fantasmas. En su texto "Sobre la 'realidad psicológica' del significado literal", Dascal dice que el significado literal "aunque sea mínima su contribución al contenido, parece desempeñar un papel crucial en el proceso de la interpretación... Esta función de control o papel dirigente quizá sea la responsable de algunas dificultades empíricas para descubrir la 'presencia' de significado literal en ciertos estudios experimentales, ya que podría ser tan sutil que difícilmente podría detectar la medida extremadamente rudimentaria del 'tiempo de reacción'" (pp. 36-7; subrayados míos). Gibbs se lanza contra esta concepción fantasmal del significado literal: "Dascal wants us to accept, almost as a matter of faith, that literal meaning exists and plays an obligatory role in comprehending speech despite the empirical evidence to the contrary. But if the guiding role that literal meaning has in linguistic processing is potentially so subtle as to be undetectable, then Dascal has essentially asked us to embrace a theory that is nearly impossible to falsify" ("Understanding and literal meaning", pp. 9-10). Pero, por otro lado, Dascal denuncia magistralmente el mismo proceso de entificación, cometido ahora por los "contextualistas" a la Gibbs: "...the problem arises in a sharper form in the case of understanding utterances in what experimenters call a 'no context' situation. In these cases, utterances are understood, and -what a coincidence!- it is their literal or direct interpretation that subjects tend to choose. Gibbs' strategy in dealing with these cases consists in assuming that even here 'context' is at work, though 'perhaps (a) so widely shared (one) that there seems to be no context at all'. Isn't this making context an entity 'so subtle as to be undetectable', just for the sake of preser- 
ving the contextualist dogma?" (Dascal, "On the Roles of Context and Literal Meaning in Understanding", p. 8). Discrepo rotundamente de Dascal en que su literalismo moderado escape de esta misma crítica. En la medida en que ambas posiciones pretenden describir conductas y descubrir entidades, cometen exactamente el mismo error lógico-conceptual: si los significados literales o los contextos son cosas, entonces ellos existen siempre... jaun alli donde no aparecen! En verdad, jellos aparecen cuando son construidos, y no aparecen cuando no lo son!

Toda la argumentación anterior está estrechamente vinculada a la concepción del "significado literal" como "invariancia", como lo "común" a emisiones. Esto quiere decir que si Dascal no acentúa esta concepción, y sí acentúa, en cambio, otras concepciones del "significado literal", el panorama de la discusión cambia sustancialmente. Me parece que ése es precisamente el caso. En "Sobre la 'realidad psicológica' del significado literal", cuando está enumerando las características de su "concepción alternativa del significado literal", él menciona que "particularmente se debe permitir la posibilidad de 'neutralización' a ciertos componentes del significado literal en algunos contextos, lo que permite contrarrestar la objeción puesta por Allwood (1981) contra la opinión de que el significado literal de una frase es aquel que es común a todos los contextos de uso" (p. 34). En su libro Pragmatics and Philosophy of Mind I. Thought in Language, a la luz de la teoría tripartita que él desarrolla allí (Introduction, 1.3), en la cual él distingue entre "sentence-meaning", "utterance-meaning" y "speaker-meaning", la segunda dimensión - "utterance-meaning"- parece ser el lugar donde se manifiesta el "significado literal", resultante de cierta influencia del contexto sobre la "sentence-meaning", y Dascal coloca el acento en la característica de convencionalidad de esa dimen- 
sión: "Utterance-meaning corresponds to what a speaker conventionally meant by this utterance..., whereas speaker's meaning may go beyond what he conventionally meant. This is why the former, but not the latter, is properly called 'semantic"' (p. 36-7). Y un poco más adelante, "... what is conventionally meant is no necessarily context-invariant" (p. 37). Y en "On the Role of Context and Literal Meaning in Understanding", explica: "Utterance-meaning is, thus, the result of a necessary attempt to complete sentence-meaning. In this sense it deserves to be viewed as the 'literal' -or, as I prefer to call it, the 'direct'- interpretation of the utterance" (p. 4). Ahora bien, todo esto forma parte de lo que he llamado, en mi artículo, una serie increíble de concesiones a los críticos modernos del "significado literal". Él concede, sucesivamente, que una expresión puede tener muchos significados literales, que el significado literal no es suficiente para interpretar oraciones, que a veces no se le puede retener para conocer la significación total, que existe una intensa interacción entre los significados literales y el entorno, cotextual y contextual, y que el significado literal, en su concepción, es tan flexible, elástico y adaptado al contexto, que admite incluso "grados de literalidad". A través de todo ese juego de concesiones, Dascal pretende hacer avanzar un programa de "reconstrucción" de esa noción, pero uno podría preguntarse en este punto cuáles son los criterios de identificación de una noción: en el caso presente, ¿con qué derecho puede llamarse aún "significado literal" a esa noción "reconstruida"? La noción dascaliana es ya tan flexible, elástica y adaptada a los contextos, que deja de poseer la característica de una categoría unificante, $\mathrm{y}$, por lo tanto, sale completamente fuera del alcance de todas las críticas de mi artículo. Dascal tiene toda la razón al decir que mis críticas no afectan su teoría de un conjunto de condicio- 
nes, ninguna de ellas necesaria, y tan flexible que permite dar cuenta de mi intuición de la "extrema diferenciación del lenguaje", pero no la afectan precisamente porque Dascal ya no defiende en absoluto una teoría del lenguaje que incluya una noción de significado literal, en un sentido inteligible de ese término. En este sentido, concuerdo plenamente con Dascal cuando afirma (en "Sobre la 'realidad psicológica'...", p. 36): "Considero bastante injusto describir esta posición - según hace Gibbs- 'no más que una defensa de la teoría tradicional'". La posición de Dascal está tan lejos de la teoría tradicional como cualquier teoría contextualista, en lo que se refiere a su problematización de lo literal.

La lucha de Dascal - a veces francamente heroicaen favor del significado literal, paradójicamente realizada a través de la problematización radical de ese concepto, está vehiculizada por el deseo de que el mismo, además de interesante, plausible o metodológicamente conveniente, sea también real. Esta ansiedad vincula la posición de Dascal, curiosamente, con cierto platonismo que él critica en otros contextos problemáticos. Por el contrario, la distinción entre las tesis $\mathrm{T} 1$ y $\mathrm{T} 2$, como fue hecha antes, trata de evitar la ontologización platónica. Diferenciar entre un plano teórico y un plano metodológico no es platonismo más que en el sentido de distinguir entre dos cosas tan diferentes como la necesidad teórica y la conveniencia metodológica o el interés psicológico o social por una noción. En lógica sentencial, sabemos que las negaciones, las conjunciones y las disyunciones son teóricamente innecesarias, una vez definido el "stroke" de Scheffer, aun cuando continuemos usándolas como abreviaturas útiles e intuitivas en nuestro trabajo cotidiano como lógicos. Esta actividad habitual no debería hacernos olvidar el valor de un resultado teórico: la dispensabilidad y superficialidad de aquellos operadores. De nin- 
guna manera puedo decir que del hecho de que el "stroke" de Scheffer alarga enormemente las expresiones lógicas, hasta tornarlas inmanejables, se siga que la negación, la conjunción y la disyunción no pueden teóricamente reducirse a él. De manera análoga, tampoco puedo decir que del hecho de que la no utilización de significados literales torne las teorías del lenguaje enormemente complejas, obligándolas a lidiar puramente con contextos infinitos, etc., se siga entonces que los significados literales son teóricamente necesarios para construir una teoría del lenguaje. Dascal confunde frecuentemente estos dos planos: "Pero, ¿qué es lo que [Cabrera] quiere decir con esa co-constitución dinámica? ¿Quizá que los términos en sí no aportan nada de propio a la constitución del significado del enunciado que componen? Si es así, habría que tratar cada enunciado como una frase hecha, y la semántica de una lengua como una lista (infinita) de los significados de tales frases. Aunque se reconozcan las limitaciones del principio de composicionalidad, sería absurdo negar de esa forma cualquier función a este principio" ("Una crítica reciente...", p. 35). Y, de nuevo, en "La 'realidad psicológica'...": "No hay duda de que la composicionalidad es pertinente para la determinación del significado: si no lo fuera, uno tendría que asumir el aprendizaje de memoria de enormes listas de interpretaciones" (p. 35). ¡Pero esto es una mezcla total de planos! La cuestión es la siguiente: si entendemos significado literal como invariancia y comunidad a través de emisiones, y si tuviéramos máquinas suficientemente rápidas y económicas como para hacer el análisis frase por frase, no habría absolutamente ningún tipo de obstáculo teórico que nos impidiese elaborar la programación de esa máquina. Los significados literales no son necesarios teóricamente: ¡nuestra finitud no puede, de ninguna manera, ser un argumento contra esa tesis! 
Lo máximo que se puede decir de un concepto cuando es legítimo (y el concepto de "significado literal" es legítimo) es que él es construible. Como he tratado de mostrar aquí, y como también Gibbs ha argurnentado en su trabajo, no creo que Dascal pueda demostrar - sin comprometerse con una teoría irrefutable, en el sentido de Popper- que hay significados literales. Pero su próximo paso podría ser mostrar que esa noción, además de construible (que es lo máximo que yo estoy dispuesto a afirmar acerca de su estatus ontológico), debe necesariamente ser construida. Para mostrar eso, Dascal debería partir de una línea de argumentación totalmente diferente de la que ha transitado hasta ahora para responder a sus adversarios -entre los cuales yo no me cuento- sumergiéndose decididamente en el problema lógico-epistemológico de la construcción de teorías. Así como Wittgenstein hablaba de la "profunda necesidad de una convención", creo que Dascal debería probar ahora "la profunda necesidad de una construcción". Sólo así, creo yo, estaría definitivamente a salvo de la nostalgia.

\section{INDICACIONES BIBLIOGRÁFICAS}

Cabrera, Julio, "Cortando árboles y relaciones (una reflexión escéptica en torno de un tema de Searle)". Crítica, México, vol. XVI, núm. 46, abril 1984.

_-, "A Lógica condenada. Uma abordagem extemporânea de filosofia da Lógica". Hucitec, São Paulo, 1987.

Dascal, Marcelo, "Pragmatics and the Philosophy of Mind I. Thought in Language". John Benjamin Publishing Company. Amsterdam-Philadelphia, 1983.

_- "Una crítica reciente a la noción de significado literal". Crítica, vol. XVIII, núm. 53, México, agosto 1986.

_- "Sobre la 'realidad psicológica' del significado literal". En Filosofía del lenguaje, de la ciencia, de los derechos humanos, y problemas de su enseñanza. Compiladores: Lourdes Valdivia y Enrique Villanueva, México, UNAM y SOFIA, 1987. 
-, "On the Roles of Context and Literal Meaning in Understanding". Cognitive Science (por publicarse).

Gibbs, Raymond W., "Understanding and Literal Meaning". Cognitive Science (por publicarse).

Recibido: 18 enero 1989. 